\title{
Making emerging phenomena a research priority
}

\author{
Manjit S. Yadav ${ }^{1}$
}

Published online: 5 February 2018

(C) Academy of Marketing Science 2018

Almost five decades ago, Phil Kotler published an intriguing article entitled "The Future of the Computer in Marketing" (Kotler 1970). Eugene Kelly, Editor of the Journal of Marketing at that time, included Kotler's article in a Special Issue that also featured contributions by several leading marketing scholars (e.g., John Howard, Paul Green, Robert Ferber, and others). From the temporal vantage point of 1970, the exploratory positioning of Kotler's article appears completely understandable - after all, the first computer with integrated circuits had appeared just a few years prior, and the use of computing technology in organizations was still in its infancy. Nevertheless, reading Kotler's article nearly five decades after its publication, I do admire the bold, broad-brush strokes with which he discussed a number of significant changes that were worthy of attention. With some degree of speculative flourish, he noted that the "marketing executive is headed toward a brave new world of instant information and sophisticated decision models" (Kotler 1970, p. 14).

Looking now at Kotler's article through the lens of our contemporary research sensibilities and evaluative criteria, one can quickly find much to critique. In fact, with the benefit of 20/20 hindsight, many readers may even be dismissive about some of the seemingly sweeping claims and the relative inadequacy of supporting evidence and arguments. As a discipline advances and standards of scholarship evolve, looking at research efforts retrospectively can sometimes result in such an evaluative stance. However, my point here is not about changing research standards per se, but rather about our discipline's diminished willingness to

Manjit S. Yadav

myadav@mays.tamu.edu

1 Mays Business School, Texas A\&M University, College Station, TX, USA operate in emerging, less-developed research areas. Our field's expectation of rigor, which is indisputably a healthy trait for any discipline, has unfortunately ossified into an inflexible view about when a research topic is considered amenable for research. In many cases, this means that only mature research topics are considered appropriate for serious research inquiry. When research topics have matured, there is usually plenty of data; these data can prove to be indispensable during the review process where authors must defend the validity of knowledge claims. However, this need to defend knowledge claims in a theorytesting context extracts a significant price from a discipline: whole swaths of the knowledge development landscape are left untouched for years before they are judged suitable for systematic inquiry.

To highlight how much our field's perspective on knowledge - and knowledge development approaches — has shifted, consider the following illustrative emerging topics related to the broad area of computing: quantum computing, machine learning, Blockchain, Internet of Things, and 3-D printing. These substantive topics, to varying degrees, exhibit this characteristic of newness that the "computer" may have had in the late 1960s when Kotler was writing the article noted earlier. Just as the computer and its implications may have appeared somewhat esoteric and ill-structured to Kotler in the late 1960s, these contemporary digital technologies are likely to engender similar reactions from marketing scholars today. In Kotler's knowledge development context several decades ago, a few exemplars had emerged in the marketplace that he could use to discuss broader, long-term implications. Similarly today, in the context of some of the emerging digital technologies noted above, we do have some early-stage examples of how firms are deploying (or could deploy) them for strategic purposes. For instance, even in the case of quantum computing - which is an early-stage and a less-understood digital technology today-there are illustrative case studies 
of initial best practices (Nicas 2017). Firms such as Google and IBM have already demonstrated, with prototypes, the exponential leap in computing prowess that can result from quantum computing. Competition for technological leadership in this area is intensifying, with significant long-term implications for related emerging areas such as machine learning that could potentially transform a whole array of activities in the marketplace.

Now do this simple thought experiment. Imagine that, inspired by Kotler's prescient 1970s article, a marketing scholar of more recent vintage decides to write about an emerging research area such as quantum computing and its implications for machine learning and subsequent marketplace activities. The difficulties involved in publishing such work in a major marketing journal, even if developed with rigor that is feasible for an emerging topic, are likely to be substantial. In fact, realistically, the odds are stacked high against a knowledge development endeavor of this type. Scholarly efforts directed at other emerging phenomena may face similar challenges. Why? Our discipline's research taste - and not just the underlying research standards - has changed dramatically over the past several decades. The discipline now prefers, or even demands, "full solutions" — there is simply little or no room for a modern version of Kotler's article.

My main contention here is that the prevailing scholarly landscape in marketing significantly limits our discipline's knowledge development endeavors and, over the long term, its knowledge output. I believe this is a serious structural impediment from the perspective of knowledge development because it removes from initial inquiry precisely those areas that are the most challenging (and potentially most impactful). When a discipline's knowledge development process fails to focus systematically on the early stages of an emerging phenomenon, it can miss key "initial conditions" that may hold important clues about underlying causal mechanisms. From a systems-theoretic perspective, the resulting knowledge development deficiencies can be significant. Although there is no simple panacea, I would like to examine some facets of this issue and propose ideas for facilitating further discussion in our field.

\section{Characteristics of emerging phenomena}

To understand the characteristics of emerging phenomena, it may be instructive to start with Merriam-Webster's definition of the word phenomenon as "an observable fact or event." In marketing, illustrative phenomena may include a broad range of substantive contexts: a new technological innovation (e.g., railroads in the nineteenth century); a new method of selling (e.g., malls in the twentieth century); or a fundamental shift in how firms and consumers communicate with one another (e.g., social media in the early part of the twenty-first century). Although the potential scope of such marketing-related developments and events is extensive, there are several questions that are applicable to all phenomena, in general. For instance: Who observed the phenomenon and saw a potential connection with marketing (e.g., managers or consumers)? Where was it observed (e.g., in the marketplace or in firms)? When was it first observed (e.g., recently or long time ago)? How was it observed (e.g., directly or as reported by others)? Answers to such questions have much significance for knowledge development in a discipline - a topic that I will return to later in this essay.

Once a phenomenon has been observed, one can say that it "exists" and thus can potentially become the subject of systematic inquiry. Therefore, a phenomenon can be viewed as having something akin to a chronological age: it comes into existence and then matures with the passage of time. During the early phase, a phenomenon can be characterized as emerging. Consistent with this perspective, Merriam-Webster describes an emerging phenomenon as "a fact or event of scientific interest susceptible to scientific description and explanation." As time passes, an emerging phenomenon can gradually evolve into a mature phenomenon.

A phenomenon that is considered relevant and worthy of inquiry by one discipline may (or may not) be of little interest to scholars in other disciplines. Brinberg and McGrath's (1985) Research Network Schema characterizes a discipline as an interlocking set of three domains: substantive (comprised of phenomena considered appropriate for study), conceptual (explanations for what is studied), and methodological (approaches employed for designing and conducting the studies). In general, every research process involves the intersection of one or more of these three domains. A typical research process often involves starting with the substantive domain (i.e., selecting a problem for study), selecting elements from the conceptual domain (e.g., identifying a pertinent theory and related concepts), and finally adopting a specific approach from the methodological domain (e.g., developing a research design for conducting an investigation). Scholars may, of course, adopt alternative research paths. An important point to note here, however, is that the substantive, conceptual, and methodological domains often shape one another. For instance, the prevalence of a specific set of methodological tools may direct attention disproportionally at certain phenomena simply by virtue of the fact that those methods are appropriate for the examination of specific phenomena. The old adage "To a hammer everything looks like a nail" underscores the potentially detrimental impact of such interactions.

\section{Challenges of emerging phenomena}

The relative newness of emerging phenomena is a double-edged sword: the newness makes such phenomena promising candidates for impactful research endeavors, but it also creates significant challenges as well. Below, I will briefly describe these challenges and provide illustrative examples from a contemporary emerging phenomenon: the digitization of markets (for additional contextual details, see Yadav and Pavlou 2014). 
Although the factors discussed below are presented as challenges, we should also view them as potential opportunities for new and innovative research (see also Table 1).

From the perspective of developing a research program on emerging phenomena, a significant challenge is the lack of attention in scholarly forums - especially during the criticallyimportant early stages. As noted above, an emerging phenomenon comes into existence only after someone observes the phenomenon and successfully makes a case that it is worthy of further scrutiny. It is through this process that a discipline establishes substantive legitimacy for a phenomenon. Academic environments, in general, have to strike a fine balance between two inherently opposing considerations: they must connect closely with the substantive domains of inquiry, but also maintain a certain degree of separation so that their knowledge development endeavors are not overly shaped by the constant shifts that may keep occurring in the substantive domains. When this balance is managed well, a discipline can keep advancing its knowledge development mission. However, if this results in isolation, it is conceivable that a number of important phenomena in the substantive domain can remain unobserved for years. Unfortunately, in the case of emerging phenomena, there is a high likelihood that the latter scenario may occur. It took decades before substantive issues pertaining to the internet and related technologies became an integral part of the marketing discipline's substantive domain.

The second challenge is that an emerging phenomenon, relative to mature phenomena, is likely to be more ill-structured. The term ill-structured, in this context, refers to the lack of clarity about several substantive issues of relevance; that is, the focal phenomenon is ill-defined. First, there is a need for clarity regarding the claim that a focal emerging phenomena is, indeed, new. That is, scholars need to delineate clearly how and why an emerging phenomenon is distinct from other phenomena (both within a discipline and across disciplines). Second, if a phenomenon is indeed new, what are the underlying characteristics that make it fundamentally distinctive? Also, what are the similarities, if any, with other phenomena? Third, an emerging phenomenon has much less clarity about key terminology that can guide scholarly discourse; it takes time for a discipline to establish such a vocabulary. The early years of the marketing literature related to the internet (i.e., in the mid-1990s) reflect scholars' efforts to establish why this new digital artifact was

Table 1 Emerging phenomena: research challenges and recommendations

\begin{tabular}{ll}
\hline Research challenges & Recommendations for facilitating research \\
\hline - Lack of attention & • Enhance observability \\
- Ill-structured & Enable early structuring \\
$\quad$ substantive domain & - Encourage initial conceptualizations \\
- Causal ambiguity & - Accelerate data availability \\
\hline
\end{tabular}

new, how it differed from previous generations of communication technology, and why there was a pressing need for new vocabulary (e.g., computer-mediated environments, social media, product migration, etc.) to facilitate further inquiry.

The third research challenge - causal ambiguity - refers to the lack of clarity about underlying conceptual mechanisms that could be operating and shaping the outcomes of an emerging phenomenon. In the early years of an emerging phenomenon, it is unclear which factors may be the definitive causal drivers of the phenomenon. A retrospective examination may provide initial insights, but this is feasible only if there are other similar (mature) phenomena that scholars can use for purposes of comparative analysis. In addition to the antecedents, there may be little or no clarity about the long-term implications of an emerging phenomenon: who may be affected, who is likely to remain unaffected, or when the expected outcomes may or may not materialize. Furthermore, without a nomological network that connects a relevant set of antecedent and outcomes constructs, a discipline is likely to struggle in its efforts to discuss casual mechanisms in a conceptually rigorous manner. The illstructured nature of the emerging phenomenon and the lack of appropriate data (more on this below) creates an ominous "perfect storm" for a discipline - the phenomenon is characterized by much uncertainty, and there is little applicable data to understand and manage this uncertainty. The resulting challenges to address causal ambiguity can thus be extremely challenging in the early years of an emerging phenomena. Case in point is the marketing discipline's efforts during the early years to understand issues such as the growth of e-commerce, impact of social media, and disruptive changes occurring in value chains.

The fourth challenge stems from data scarcity. Emerging phenomena, by definition, are new and thus there is little or no data available during the early years. Therefore, such phenomena are often (but not always) data deficient. As scholars develop consensus about underlying terminology (i.e., what to measure) and methods (i.e., how to measure), the initial years of inquiry can present significant challenges. The lack of data, in turn, can often complicate attempts to understand an emerging phenomenon's growth, the entities that play a role in spurring this growth, and the broader long-term implications of that phenomenon. It is like driving blindfolded - with all the attendant dangers and vulnerabilities. In the case of the internet, the underlying technological developments commenced in late 1960s. However, when marketing scholars started systematic inquiry in the mid-1990s, there was almost no directly applicable data about firms, consumers, and the emerging digital marketplace. Now, more than two decades later, the advent of "Big Data" has replaced data scarcity with abundance. In fact, this relative abundance of data now demands increased selectivity, discipline, and innovativeness from scholars. This dramatically transformed data situation - a veritable embarrassment of riches - is in stark contrast with the data deficient environment that prevailed in the early years. 


\section{Facilitating research on emerging phenomena}

As discussed above, emerging phenomena have certain inherent characteristics that can create substantial impediments for a discipline's efforts to study and understand that phenomenon. However, a discipline's ability to study emerging phenomena effectively and efficiently is critically important for its long-term health and impact. Like "ambidextrous" organizations that are simultaneously able to work on established and nascent opportunities (O'Reilly and Tushman 2013), I believe that disciplines must develop similar capabilities in the realm of knowledge development. But how? Although a comprehensive treatment of such a complex topic is beyond the scope of this essay, I will conclude with a few thoughts for the marketing discipline. My overarching goal is to spur further debate and discussion on a number of imperatives that need attention. In the context of these imperatives, I also propose a few "action items" for moving forward.

The first imperative is to enhance the observability of emerging phenomena. As noted above, a phenomenon comes into existence only when someone observes it. Interesting, new phenomena can remain "under the radar" for a long duration if our discipline does not have appropriate mechanisms that increase the likelihood of their early detection. This, of course, raises the question: How does our discipline monitor shifts in its substantive domain? Although several formal and informal mechanisms could be at work here, both experienced and new scholars are likely to be the key actors in these mechanisms; they are the eyes and ears of the discipline. To increase the likelihood that these actors do, in fact, succeed in detecting an early-stage signal in the substantive environment, we need to create more immersive observational opportunities for them. This can be accomplished via several initiatives such as: (1) adding a higher level of substantive relevance and vitality to our professional conferences; (2) creating more frequent and meaningful dialogs with knowledgeable managers who have a unique, front-line seat in the marketplace; (3) encouraging doctoral students to develop a comprehensive understanding of the marketing discipline's substantive scope and its longterm evolution; (4) and, finally, crafting a more ambitious role for organizations such as the Marketing Science Institute to ensure that our discipline is able to spot early-stage phenomena in a more timely manner.

The second imperative is to enable early structuring of emerging phenomena. As noted earlier, emerging phenomena tend to be ill-structured due to the lack of clarity regarding terminology and substantive distinctiveness. To address this issue, we should encourage research efforts that clarify new terminology that may be relevant in the context of an emerging phenomena. Introducing such terminology during the early years of an emerging phenomenon allows the discipline's scholarly community to develop pertinent vocabulary that facilitates ongoing dialog and additional research initiatives. Once there is clarity about terminology, research efforts can create typologies that juxtapose an emerging phenomenon with other more established phenomena with which our discipline has a higher level of prior familiarity. In-depth historical analyses and integrative reviews, by providing a longitudinal context, can also be invaluable in this regard (Palmatier et al. 2018). Of course, the value of such research efforts is likely to vary; for radically new emerging phenomena, comparisons with prior phenomena will have limited utility. Nevertheless, as a general strategy, our discipline should encourage such research efforts - especially if the authors can go beyond a descriptive stance and demonstrate value for ongoing theory development.

The third imperative is to encourage initial conceptualizations that alleviate, at least partly, causal ambiguity about a focal emerging phenomenon. A good starting point in this regard is the exploration and identification of concepts. Depending on the initial structuring work that has been done (see above), scholars may be in a position to identify and develop concepts that have explanatory ability. These concepts do not always have to be completely new; it may be feasible (and even desirable) sometimes to repurpose existing concepts to shed light on selected aspects of a new phenomenon. Once a set of pertinent concepts has been identified, logic and literature-based research efforts-including analytical models - can be aimed at proposing an initial nomological network that has potential explanatory value. Specialized journals such as AMS Review that are focused exclusively on conceptual contributions aimed at theory development, in collaboration with other more broadly-positioned journals in our discipline, can facilitate such research efforts-from concept development to proposing an initial nomological network of relevant constructs.

The fourth imperative is to accelerate data availability about emerging phenomena so that the discipline makes progress efficiently and effectively toward theory testing. To establish the validity of scientific knowledge claims, appropriate data are essential. As noted earlier, emerging phenomena are often (but not always) data deficient; even when data are readily available, it will take time to have access to rich longitudinal data as the emerging phenomena evolves. Given these challenges, our discipline must actively cultivate relationships with entities that can help source data in an expedited manner. This can be accomplished with individual corporate partners, or with the assistance of a consortium of firms who may wish to pool data. In previous decades, initiatives such as PIMs (Profit Impact of Market Strategy) served as a very useful resource for studying mature phenomena. What our discipline needs now is a new generation of well-funded initiatives that are focused on sourcing and disseminating data for facilitating research on emerging phenomena. In this regard, research centers can play an invaluable role (see, e.g., Kumar 2017). 
More generally, to facilitate the specific initiatives noted above, we need to create and strengthen a disciplinary culture of innovation with respect to its knowledge development efforts. The marketing discipline has undoubtedly progressed on many fronts, but our knowledge development approaches have increasingly become scripted - a limited number of successful templates for knowledge development have emerged and they dominate the way we think about scholarship. We need to find a way to break away from the straightjacket-like grip of the prevailing templates of knowledge development (Lynch et al. 2012; Yadav 2010). We need a renewed emphasis on imagination (see Zaltman 2016), an infusion of new ideas, and above all, intellectual courage to expand our repertoire of knowledge development approaches. This will necessitate a critical rethinking of all aspects of our discipline's knowledge development approach —our journals, our institutions, our incentive systems, and how we train the next generation of scholars.

Although a multi-pronged approach is essential, the critical role played by our journals is worth emphasizing because they are the most visible and impactful agents of change in the discipline. There are some initial encouraging signs in this regard-for example, Marketing Science's new section, Frontiers of Marketing Science, that seeks to "accelerate entry of new ideas." It is a noteworthy endeavor, but much more structural innovation is needed at all journals so that we can fully harness the creative energy of scholars in our discipline. Looking ahead, additional experimentation with carefully curated new sections - each with a distinct editorial mission - is certainly one mechanism that holds much promise. Consider, for instance, the highly influential New England Journal of Medicine that features an intriguing array of sections: Original Research, Perspective, Review Article, Sounding Board, etc. Such sections, selected and developed judiciously, can facilitate a rigorous and invigorating scholarly environment in our discipline - and address a number of issues noted in this essay. Undoubtedly, creating and effectively managing such structural changes in journals is a Herculean task of considerable complexity. However, with institutional support and a deepseated determination to bring about change, it can be done.

Emerging phenomena represent a significant knowledge development opportunity for the marketing discipline. The timely and effective examination of such phenomena lies at the heart of our discipline's dynamism and long-term impact. However, the inherent characteristics of such phenomena can create significant impediments from the perspective of research. To address these impediments, I have outlined in this essay several imperatives to facilitate further discussion. Over time, through this process of reflection and communication, I hope that impactful new ideas will emerge that will further strengthen our discipline.

Acknowledgements I would like to thank Rob Palmatier for the invitation to develop this Guest Editorial. Len Berry, Bernie Jaworski, Ajay Kohli, Debbie MacInnis, Rajan Varadarajan and Jerry Zaltman provided helpful comments on an earlier draft.

\section{References}

Brinberg, D. L., \& McGrath, J. (1985). Validity and the research process. Beverly Hills: Sage Publications.

Kotler, P. (1970). The future of the computer in marketing. Journal of Marketing, 34(January), 11-14.

Kumar, V. (2017). The role of university centers in promoting research. Journal of the Academy of Marketing Science, 45(4), 453-458.

Lynch, J. G., Alba, J. W., Krishna, A., Morwitz, V. G., \& Gürhan-Canli, Z. (2012). Knowledge creation in consumer research: Multiple routes, multiple criteria. Journal of Consumer Psychology, 22(4), $473-485$.

Nicas, J. (2017). Welcome to the quantum age. The Future of Everything. Supplement to the Wall Street Journal, November/December 2017, 60-67.

O'Reilly, C. A., \& Tushman, M. L. (2013). Organizational ambidexterity: Past, present, and future. Academy of Management Perspectives, 27(4), 324-338.

Palmatier, R. W., Houston, M. B., \& Hulland, J. (2018). Review articles: purpose, process, and structure. Journal of the Academy of Marketing Science, 46(1), 1-5.

Yadav, M. S. (2010). The decline of conceptual articles and implications for knowledge development. Journal of Marketing, 74(January), 119.

Yadav, M. S., \& Pavlou, P. A. (2014). Marketing in computer-mediated environments: Research synthesis and new directions. Journal of Marketing, 78(January), 20-40.

Zaltman, G. (2016). Marketing's forthcoming age of imagination. AMS Review, 6(3-4), 99-115. 